\title{
Repetitive Transcranial Magnetic Stimulation for Chronic Prostatitis/ Chronic Pelvic Pain Syndrome: A Prospective Pilot Study
}

\author{
Jussi Nikkola ${ }^{1,2}$, Anu Holm ${ }^{3,4}$, Marjo Seppänen ${ }^{1}$, Teemu Joutsi ${ }^{1}$, Esa Rauhala ${ }^{3}$, Antti Kaipia ${ }^{2}$ \\ ${ }^{1}$ Department of Urology, Tampere University Hospital, Tampere, Finland \\ ${ }^{2}$ Department of Surgery, Satakunta Hospital District, Pori, Finland \\ ${ }^{3}$ Unit of Clinical Neurophysiology, Satakunta Hospital District, Pori, Finland \\ ${ }^{4}$ Faculty of Health and Welfare, Satakunta University of Applied Sciences, Pori, Finland
}

Purpose: To evaluate the feasibility, efficacy, and safety of repetitive transcranial magnetic stimulation (rTMS) in patients with treatment-resistant chronic prostatitis/chronic pelvic pain syndrome (CP/CPPS).

Methods: Eleven patients with CP/CPPS were enrolled in this prospective clinical study. rTMS was performed for 5 consecutive days in 20-minute sessions. Patients were evaluated at baseline, after treatment, and at 1, 4, 8, and 12 weeks after the last session with questionnaires concerning pain (numerical rating scale [NRS], the National Institutes of Health Chronic Prostatitis Symptom Index [NIH-CPSI], and the Short Form-36 [SF-36]), urinary symptoms (NIH-CPSI, Danish Prostatic Symptom Score [DAN-PSS-1]), quality of life (NIH-CPSI, SF-36), and psychometrics (Beck Depression Index [BDI]). Telephone-based interviews were used to evaluate side effects, subjective response, and changes in drug consumption.

Results: All patients completed the planned treatment and follow-up according to protocol. No patients experienced serious side effects or significant pain increase during or after treatment. Mild transient tension headache responsive to oral pain medication was reported by 2 patients. Decreased pain was observed on the NRS after treatment and at 1 and 8 weeks $(\mathrm{P}=0.019, \mathrm{P}=0.006, \mathrm{P}=0.042$, respectively) and on the NIH-CPSI pain domain at 1 week $(\mathrm{P}=0.04)$. Improvement in lower urinary tract symptoms was observed after treatment in the NIH-CPSI urinary domain $(\mathrm{P}=0.02)$ but not with the DANPSS-1. No significant changes in the BDI were observed. Nine patients reported a positive overall subjective response (82\%) and 6 patients $(55 \%)$ were able to reduce pain medication. Higher age was associated with lower NRS scores after treatment $(\mathrm{R}=0.605, \mathrm{P}=0.048)$ and at 8 weeks $(\mathrm{R}=0.659, \mathrm{P}=0.028)$.

Conclusions: rTMS for patients with CP/CPPS seemed to be well tolerated, at least moderately effective in pain reduction, and might be of interest in patients with chronic pelvic pain resistant to conventional treatment. These findings remain to be confirmed by a randomized trial.

Keywords: Chronic prostatitis; Chronic pelvic pain syndrome; Transcranial magnetic stimulation; Pain management; Lower urinary tract symptoms

- Research Ethics: The study was performed according to the Helsinki Declaration and approved by the Medical Ethics Committee of Turku University Hospital (137/1801/2017). All patients provided written informed consent prior to participation in the study.

- Conflict of Interest: No potential conflict of interest relevant to this article was reported.

Corresponding author: Jussi Nikkola (iD https://orcid.org/0000-0002-5077-2012 Department of Urology, Tampere University Hospital, Kuntokatu 2, 33520, Tampere, Finland

E-mail: jussi.nikkola@fimnet.fi

Submitted: December 26, 2019 / Accepted after revision: March 16, 2020
This is an Open Access article distributed under the terms of the Creative Commons Attribution Non-Commercial License (https://creativecommons.org/licenses/by-nc/4.0/) which permits unrestricted non-commercial use, distri-
bution, and reproduction in any medium, provided the original work is properly cited. 


\section{INTRODUCTION}

Chronic prostatitis $(\mathrm{CP})$ is common among men, with a lifetime prevalence of $10 \%-14 \%[1,2]$. The most common form is type III CP or chronic prostatitis/chronic pelvic pain syndrome (CP/CPPS) [3]. Urogenital pain, which is typically the most severe symptom of CP/CPPS, can severely diminish patients' quality of life (QoL) and sexual function [2,4]. Lower urinary tract symptoms (LUTS) and psychological issues are also common in patients with CP/CPPS [5-7]. The standard treatment consists of trials with anti-inflammatory drugs, alpha-blockers, 5-alpha reductase inhibitors, antibiotics, antidepressants or neuromodulating drugs, and physiotherapy [8]. Despite a multimodal treatment approach, many patients have persistent symptoms [9].

In transcranial magnetic stimulation (TMS), the brain cortex is stimulated by a magnetic coil held above the cranium. Repetitive TMS (rTMS) has been used in patients with neuropathic pain due to complex regional pain syndrome, spinal cord injuries, trigeminal nerve lesions, phantom pain, as well as in patients with nonneuropathic pain (migraine, lumbar pain, visceral pain, or postoperative pain) and as a treatment of depression and tinnitus [10-12]. A recent study also showed that rTMS alleviated neuropathic pain and might improve urinary function in patients with bladder pain syndrome/interstitial cystitis (BPS/ IC) [13].

The lack of treatment options for patients with CP/CPPS raises the need for new treatment modalities. Our aim was to investigate whether rTMS is effective, feasible, and safe in CP/ CPPS patients.

\section{MATERIALS AND METHODS}

\section{Patients}

Eleven patients with treatment-resistant CP/CPPS were enrolled as participants. CP/CPPS was diagnosed based on typical symptoms of discomfort or pain resistant to standard therapies in the pelvic region for at least a 3-month period within the previous 6 months, careful clinical examination with urodynamic, laboratory, and urinalysis testing, and exclusion of other etiologies for symptoms. Patients were considered resistant to conventional treatment if they had previously received treatment with available conventional therapies including alphablockers, 5-alpha reductase inhibitors, antibiotics, nonsteroidal anti-inflammatory drugs and pain threshold-lowering drugs.
The duration of ongoing symptoms for inclusion in the study was at least 3 months, and no new medications should have been initiated during the prior 30 days. The cutoff score of the National Institutes of Health Chronic Prostatitis Symptom In$\operatorname{dex}$ (NIH-CPSI) for inclusion was $>8$ for pain and $>11$ for total points. The exclusion criteria included a recent cerebrovascular event ( $<6$ months), a cardiac pacemaker, an inner ear transplant, a medical pump, and metallic clips or bodies in the head region. Patients who had recently ( $<3$ months) undergone a prostate procedure were not included in the study. Epilepsy and bipolar disorder were considered relative exclusion criteria.

The study was performed according to the Helsinki Declaration and approved by the Medical Ethics Committee of Turku University Hospital. All patients provided written informed consent prior to participation in the study.

\section{Repetitive TMS}

Navigated rTMS treatment was performed with a Visor2 navigation system (ANT Neuro, Berlin, Germany) and a MagStim Rapid2 stimulator (Magstim Co., Whitland, Wales, UK) with an air-cooled figure-of-eight coil giving biphasic pulses. Prior to treatment, head magnetic resonance imaging was done for all patients with a 1.5-T Siemens Aera (Siemens, Erlangen, Germany) with a T1-weighted 3-dimensional sequence.

The resting motor threshold (RMT) was determined as recommended by the International Federation of Clinical Neurophysiology [14]. Motor evoked potentials (MEPs) were recorded from the left-hand thenar muscles. MEP response was defined as a MEP with a $>50-\mu \mathrm{V}$ peak-to-peak amplitude. The maximum-likelihood threshold-tracking algorithm was used to determine the TMS intensity that yielded a $50 \%$ probability of evoking a MEP [15]. The stimulation was done with the same coil that was used in the treatment sessions.

Patients received navigated rTMS over 5 consecutive days in daily 20-minute sessions, during which 750 pulses were applied over both the left and right motor cortexes at locations corresponding to the pelvic area. The coil handle was pointing backwards and parallel to the midline. In total, 1,500 pulses were delivered at trains of $10 \mathrm{~Hz}$ for 5 seconds with 26-second intertrain intervals at an intensity of $110 \%$ of the RMT. All patients received 5 full sessions and used ear plugs during the stimulation. The rTMS schedule was based on evidence-based guidelines to treat neuropathic pain using high-frequency $(10 \mathrm{~Hz})$ rTMS on the M1 motor cortex [11]. 


\section{Response and Safety Evaluation}

The primary endpoints were a decrease in the NIH-CPSI score, a decrease in mean pain experienced from the prior week using a numerical rating scale (NRS), a decrease in the score on the 36-Item Short Form Survey Instrument (SF-36) painlessness domain, a decrease in drug consumption for pain, and safety (reported adverse events) of rTMS. The secondary endpoints were effects on QoL and LUTS.

The NIH-CPSI is a questionnaire designed for $\mathrm{CP} / \mathrm{CPPS}$ that has been validated in Finnish [16]. Its 3 domains include pain (Q1-4), voiding (Q5-6), and QoL (Q7-9). The maximum total score of the NIH-CPSI is 43 points.

LUTS were more carefully assessed using the Danish Prostatic Symptom Score (DAN-PSS-1). The SF-36 was used to assess changes in various domains concerning QoL. The Beck Depression Index (BDI) was used to evaluate psychological changes.

Patients were contacted by telephone at 1, 4, 8, and 12 weeks to assess possible adverse events, subjective treatment response, and changes in the medication needed for pain control.

\section{Statistical Analysis}

The paired-samples t-test was used to analyze the effect of rTMS at different time points compared to baseline. The Fisher exact test, Mann-Whitney U-test, and Student t-test were used for bivariate comparisons analyzing factors associated with overall benefits and decreased drug consumption. Spearman correlation coefficients were used to study the effects of different variables on decreases in NRS and NIH-CPSI score. P-values $<0.05$ were considered to indicate statistical significance. The analysis was carried out using IBM SPSS Statistics ver. 22.0 (IBM Co., Armonk, NY, USA).

\section{RESULTS}

The mean age of patients was $54.3 \pm 15.9$ years, and the patients had experienced symptoms of CP/CPPS for a median of 9.2 years (range, 1.7-48.0 years). The mean NRS for pain from the prior week was $7.5 \pm 2.0$ at baseline. Demographic and clinical characteristics, as well as clinical data at baseline, are presented in Table 1. Four patients (36\%) needed daily opioid-based medication and 5 patients (45\%) were currently using neuromodulating medications (pregabalin, gabapentin, amitriptyline, or nortriptyline).

rTMS treatment was performed between March 2018 and April 2019. All 11 enrolled patients completed the 5-day rTMS
Table 1. Demographic characteristics and clinical data at baseline $(\mathrm{n}=11)$

\begin{tabular}{lc}
\hline Characteristic & Value \\
\hline Sex, male:female & $11: 0$ \\
Age $(\mathrm{yr})$ & $54.3 \pm 15.9$ \\
Body mass index $\left(\mathrm{kg} / \mathrm{m}^{2}\right)$ & $26.9 \pm 3.5$ \\
Smoking & $3(27)$ \\
Prior transurethral prostate procedure & $5(46)$ \\
Disease duration (yr) & $9.2(1.7-48.0)$ \\
Duration of ongoing symptoms (yr) & $2.3(0.4-20.4)$ \\
NRS for pain (average from prior week) & $7.5 \pm 2.0$ \\
NIH-CPSI total (max 43) & $30.3 \pm 8.0$ \\
NIH-CPSI pain (max 21) & $15.0 \pm 3.5$ \\
NIH-CPSI urinary symptoms (max 10) & $6.3 \pm 3.3$ \\
NIH-CPSI QoL (max 12) & $8.9 \pm 2.3$ \\
Beck Depression Index & $12(0-43)$ \\
DAN-PSS-1 total & $15(0-48)$ \\
\hline
\end{tabular}

Values are presented as mean \pm standard deviation, number (\%), or median (range).

NRS, numerical rating scale; NIH-CPSI, National Institutes of Health Chronic Prostatitis Symptom Index; DAN-PSS-1, Danish Prostatic Symptom Score.

protocol and the 12-week follow-up. All patients tolerated the full rTMS protocol and none of the patients reported an increase in pain assessed with NRS relative to their status before treatment. No serious side effects were reported during treatment or follow-up. Two patients reported mild headache, 1 with and 1 without neck pain, at the week 1 telephone interview. The pain was tolerable and responded to anti-inflammatory medications.

\section{Factors Associated With Responses}

A subjective positive total response to the treatment regimen was reported by 9 patients (82\%) after follow-up was completed. Six patients (55\%) were able to decrease their pain medication consumption during follow-up. One patient was able to reduce gabapentin medication from a daily dose of $300 \mathrm{mg}$ to none, 2 patients were able to reduce their daily opioid-based medication use, and 5 patients were able to reduce or discontinue daily paracetamol or nonsteroidal anti-inflammatory drugs.

Duration of symptoms, age, smoking habits, body mass index, baseline NIH-CPSI scores, baseline NRS, baseline BDI scores, and ongoing usage of neuromodulating medication 


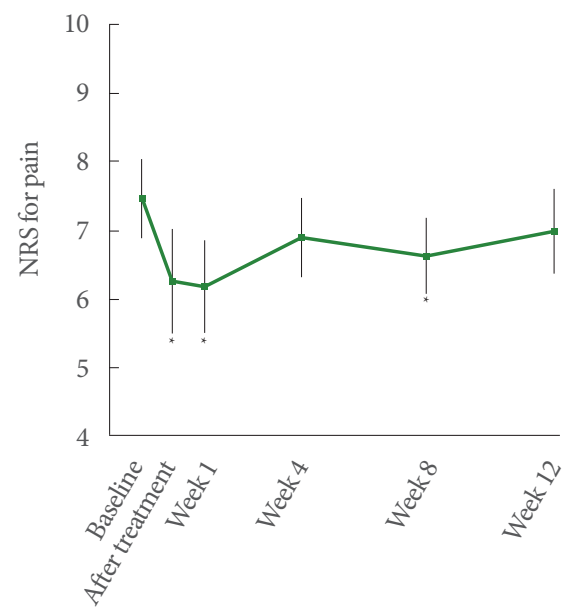

Fig. 1. Mean changes in the numerical rating scale (NRS) for pain at different time points. A significant reduction in pain was observed after treatment and at 1 and 8 weeks after treatment when compared to baseline, using the paired-samples t-test, with decreases of $1.2,1.4$, and 0.8 points respectively.

were not associated with a positive response. A decrease in drug usage during follow-up was associated with a lower baseline NRS $(P=0.079)$ and a longer duration of symptoms $(P=0.068)$.

\section{Impact on Pain}

A significant decrease in pain assessed with the NRS was observed after the treatment regimen (1.2 points, $\mathrm{P}=0.019$ ), at 1 week (1.4 points, $\mathrm{P}=0.006)$, and at 8 weeks $(0.8$ points, $\mathrm{P}=0.042)$ when compared to the baseline NRS (Fig. 1). The pain domain score on the NIH-CPSI questionnaire decreased from a mean of 15.0 points at baseline to 13.2 points $(\mathrm{P}=0.066)$ after the treatment and to 12.9 points $(\mathrm{P}=0.037)$ at 1 week (Fig. 2). The total NIH-CPSI score decreased from 30.3 points at baseline to 26.2 points $(\mathrm{P}=$ $0.021)$ after the treatment and to 27.2 points $(\mathrm{P}=0.056)$ at 1 week, after which it slowly increased to 28.7 points at 12 weeks. Regarding the SF-36 questionnaire, no significant changes in the painlessness domain were detected.

Older age was correlated with a greater decrease in the NRS during follow-up after the treatment $(\mathrm{R}=0.605, \mathrm{P}=0.048)$ and at 8 weeks $(\mathrm{R}=0.659, \mathrm{P}=0.028)$. Concurrent usage of opioids or neuropathic pain medication was not associated with changes in NRS or NIH-CPSI points.

\section{Effect on LUTS}

The NIH-CPSI urinary domain score decreased from 6.3 to 4.9 points from baseline to after treatment (1.4-point decrease,

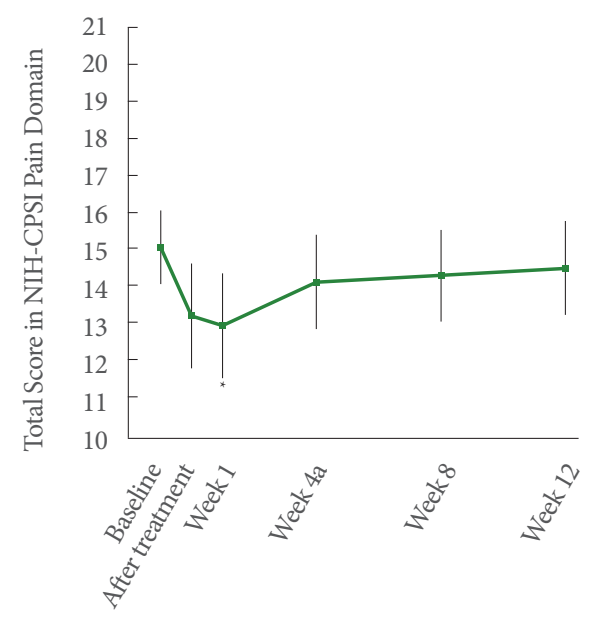

Fig. 2. Mean changes in the total score in the National Institutes of Health Chronic Prostatitis Symptom Index (NIH-CPSI) pain domain (Q1-4 in the NIH-CPSI questionnaire, maximum points 21) at different time points show a significant response in pain reduction at 1 week when compared to baseline (2.1-point decrease, $\mathrm{P}=0.037$ ).

$\mathrm{P}=0.02$ ), but no statistically significant decreases were subsequently found. No significant changes in scores on the DANPSS-1 questionnaire were detected.

\section{Effect on QoL}

The SF-36 questionnaire showed no statistically significant changes in any domain during follow-up.

\section{DISCUSSION}

In this pilot study, rTMS proved to be a safe and well-tolerated potential treatment method for CP/CPPS patients, and it may be used to alleviate pain that has been resistant to conventional therapies. Like many patients with CP/CPPS, all of the patients in this study were severely symptomatic, with pain negatively affecting their QoL despite the previous application of standard conventional therapy methods (as per the inclusion criteria). Due to the lack of effective treatment methods for CP/CPPS, these findings are of considerable interest.

The pathophysiology of CP/CPPS remains unknown and is most likely multifactorial [8]. Regardless of the trigger of the inflammatory process behind CP/CPPS (microbiological, autoimmune-based, or involving chemical or mechanical irritation caused by urine reflux), the condition leads to tissue swelling, tissue hypoxia, and tissue damage caused by inflammatory mediators (interleukins, cytokines, and histamine) [17]. Pain be- 
comes chronic due to constant inflammation and in long-lasting cases may be considered neuropathic [18]. This kind of neurogenic inflammation sensitizes mast cells to produce inflammatory mediators, leading to constant activation in nociceptive Ctype nerve fibers [17]. Nonetheless, cyclo-oxygenase inhibitors and tricyclic antidepressants, novel neuromodulating drugs (gabapentin, pregabalin), and even oral or intraprostatic corticosteroid treatment - alone or in combination - often fail.

rTMS treatment modulates intracortical inhibitory circuits and may help to repair intracortical inhibition in patients with neuropathic pain. The pain-decreasing effect of rTMS is thought to be mediated via subcortical neural networks as a result of enhancement of the dopamine-opioid system, although rTMS treatment has also been reported to enhance serum beta-endorphin concentrations [11,19-21].

Our findings are in line with 2 recent studies of rTMS on female patients. Pinot-Monange et al. [22] studied the effect of rTMS on chronic pelvic pain due to endometriosis and showed a maximum of a 1-point decrease in visual analogue scale (VAS) scores at 28 days of follow-up. In a study by Cervigni et al. [13], rTMS significantly decreased VAS scores in patients with BPS/IC, while sham treatment showed no significant effect on any parameters. The latter study also found significant improvements in BPS/IC patients' voiding parameters. In our study on male patients, no significant changes after treatment were observed in urination. This is potentially due to fact that pain in $\mathrm{CP} / \mathrm{CPPS}$ originates from the prostate or adjacent structures (pelvic floor) potentially resulting in some functional obstruction and therefore changes in bladder detrusor muscle and pelvic floor musculature; even though pain may be alleviated, changes in urination would probably appear far later. In our study, pelvic pain was the main concern of all of the patients, rather than urinary symptoms. Significant changes in QoL were also not seen in our study. Nonetheless, the performance of the SF-36 for the evaluation of CP/CPPS patients has not been studied. The most common side effect of rTMS, tension headache, has been reported in $5 \%-10 \%$ in patients, which aligns with the finding that 2 of the 11 patients in the present study reported this side effect [23].

Repeated rTMS stimulation cycles might improve synaptic strengthening [11]. We chose to investigate the effect of a single 5-day rTMS session cycle, since it would be more feasible for patients. Furthermore, according to a study by Cervigni et al. [13], even a single 5-day cycle of rTMS was associated with a positive response at 3 weeks after treatment and the response was similar to that observed in our study, with the achievement of at least a minimal clinically important difference (over a 1-point decrease in pain) [24]. Providing repeated rTMS sessions might still improve the total effect of the treatment. However, it is interesting that the NRS increased at 4 weeks of follow-up, but again significantly decreased at 8 weeks. At 12 weeks, the NRS again ascended to near the baseline level, which fits the typical response profile of rTMS and most likely indicates the need for repeated stimulation [25].

Although the duration of symptoms did not correlate to responses in this study, it is notable that these patients had experienced symptoms for a median of 9.2 years. It is possible that rTMS given earlier in the course of the disease might be associated with an even more potent response. Still, rTMS should be reserved only for patients who have persistent symptoms despite trials with standard medical therapies.

This study is limited by the lack of a control group and a limited sample size. The follow-up time was probably long enough to assess the effects of rTMS, since the effect of a single session typically lasts for under 3 months. In pain studies, the placebo effect is always a possible contributor to pain alleviation. However, it is notable that no significant changes in the BDI were observed, indicating that the clinical alleviation of pain was not due to improvements in patients' psychological status.

In conclusion, rTMS was found to be well tolerated by $\mathrm{CP} /$ CPPS patients and at least moderately effective in pain alleviation in patients refractory to medical treatments. This feasibility pilot study encourages future trials to assess and confirm the results in a randomized controlled trial setting.

\section{AUTHOR CONTRIBUTION STATEMENT}

- Conceptualization: JN, AH, MS, TJ, ER, $A K$

- Formal analysis: $J N, A K$

- Methodology: JN, $A H, E R, A K$

- Project administration: $J N, E R, A K$

-Writing - original draft: JN

-Writing - review \& editing: JN, $A H, M S, T J, E R, A K$

\section{REFERENCES}

1. Mehik A, Hellström P, Lukkarinen O, Sarpola A, Järvelin M. Epidemiology of prostatitis in Finnish men: a population-based crosssectional study. BJU Int 2000;86:443-8.

2. Franco J, Tirapegui F, Garrote V, Vietto V. Interventions for treating 
chronic prostatitis/chronic pelvic pain syndrome (Protocol). Cochrane Database Syst Rev 2016;CD012320.

3. Nickel JC, Nyberg LM, Hennenfent M. Research guidelines for chronic prostatitis: consensus report from the first National Institutes of Health International Prostatitis Collaborative Network. Urology 1999;54:229-33.

4. Walz J, Perrotte P, Hutterer G, Suardi N, Jeldres C, Bénard F, et al. Impact of chronic prostatitis-like symptoms on the quality of life in a large group of men. BJU Int 2007;100:1307-11.

5. Pavone-Macaluso M. Chronic prostatitis syndrome: a common, but poorly understood condition. Part I. EAU-EBU Update Series 2007;5:1-15.

6. Kwon JK, Chang IH. Pain, catastrophizing, and depression in chronic prostatitis/chronic pelvic pain syndrome. Int Neurourol J 2013;17:48-58.

7. Schaeffer AJ. Epidemiology and demographics of prostatitis. Andrologia 2003;35:252-7.

8. Engeler DS, Baranowski AP, Dinis-Oliveira P, Elneil S, Hughes J, Messelink EJ, et al. The 2013 EAU guidelines on chronic pelvic pain: is management of chronic pelvic pain a habit, a philosophy, or a science? 10 years of development. Eur Urol 2013;64:431-9.

9. Nickel JC, Downey J, Ardern D, Clark J, Nickel K. Failure of a monotherapy strategy for difficult chronic prostatitis/chronic pelvic pain syndrome. J Urol 2004;172:551-4.

10. Cruccu G, Aziz TZ, Garcia-Larrea L, Hansson P, Jensen TS, Lefaucheur JP, et al. EFNS guidelines on neurostimulation therapy for neuropathic pain. Eur J Neurol 2007;14:952-70.

11. Lefaucheur JP, André-Obadia N, Antal A, Ayache SS, Baeken C, Benninger DH, et al. Evidence-based guidelines on the therapeutic use of repetitive transcranial magnetic stimulation (rTMS). Clin Neurophysiol 2014;125:2150-206.

12. Lan L, Zhang X, Li X, Rong X, Peng Y. The efficacy of transcranial magnetic stimulation on migraine: a meta-analysis of randomized controlled trails. J Headache Pain 2017;18:86.

13. Cervigni M, Onesti E, Ceccanti M, Gori MC, Tartaglia G, Campagna $G$, et al. Repetitive transcranial magnetic stimulation for chronic neuropathic pain in patients with bladder pain syndrome/ interstitial cystitis. Neurourol Urodyn 2018;37:2678-87.
14. Groppa S, Oliviero A, Eisen A, Quartarone A, Cohen LG, Mall V, et al. A practical guide to diagnostic transcranial magnetic stimulation: report of an IFCN committee. Clin Neurophysiol 2012;123:858-82.

15. Awiszus F. TMS and threshold hunting. Suppl Clin Neurophysiol 2003;56:13-23.

16. Leskinen MJ, Mehik A, Sarpola A, Tammela TL, Järvelin MR. The Finnish version of The National Institutes Of Health Chronic Prostatitis Symptom Index correlates well with the visual pain scale: translation and results of a modified linguistic validation study. BJU Int 2003;92:251-6.

17. Pontari MA, Ruggieri MR. Mechanisms in prostatitis/chronic pelvic pain syndrome. J Urol 2004;172:839-45.

18. Doiron RC, Nickel JC. Management of chronic prostatitis/chronic pelvic pain syndrome. Can Urol Assoc J 2018; 12 (6 Suppl 3):S161-3.

19. Hoogendam JM, Ramakers GM, Di Lazzaro V. Physiology of repetitive transcranial magnetic stimulation of the human brain. Brain Stimul 2010;3:95-118.

20. Lefaucheur JP. Cortical neurostimulation for neuropathic pain: state of the art and perspectives. Pain 2016;157 Suppl 1:S81-9.

21. Lamusuo S, Hirvonen J, Lindholm P, Martikainen IK, Hagelberg N, Parkkola R, et al. Neurotransmitters behind pain relief with transcranial magnetic stimulation - positron emission tomography evidence for release of endogenous opioids. Eur J Pain 2017;21:1505-15.

22. Pinot-Monange A, Moisset $X$, Chauvet $P$, Gremeau AS, Comptour A, Canis M, et al. Repetitive Transcranial Magnetic Stimulation Therapy (rTMS) for Endometriosis Patients with Refractory Pelvic Chronic Pain: A Pilot Study. J Clin Med 2019;8:508.

23. Rossi S, Hallett M, Rossini PM, Pascual-Leone A; Safety of TMS Consensus Group. Safety, ethical considerations, and application guidelines for the use of transcranial magnetic stimulation in clinical practice and research. Clin Neurophysiol 2009;120:2008-39.

24. Salaffi F, Stancati A, Silvestri CA, Ciapetti A, Grassi W. Minimal clinically important changes in chronic musculoskeletal pain intensity measured on a numerical rating scale. Eur J Pain 2004;8:283-91.

25. Hamid P, Malik BH, Hussain ML. Noninvasive transcranial magnetic stimulation (TMS) in chronic refractory pain: a systematic review. Cureus 2019;11:e6019. 